\title{
Epidemiology of hospitalizations due to pesticide intoxication-associated acute kidney injury in China
}

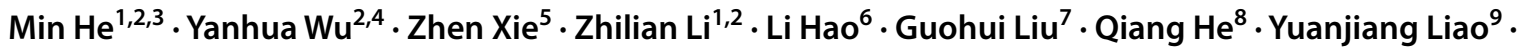 \\ Rizwangul Ghappar ${ }^{10} \cdot$ Hongwei Peng $^{11}$. Jinglie Xie ${ }^{12} \cdot$ Xiaohong Wei $^{13} \cdot$ Yusheng Zhang $^{14}$. Qiongyi Huang ${ }^{1}$. \\ Yuanhan Chen ${ }^{1,2} \cdot$ Xinling Liang ${ }^{1,2}$ on behalf of China collaborative study on AKI (CCS-AKI)
}

Received: 18 December 2020 / Accepted: 8 May 2021 / Published online: 18 May 2021

(c) The Author(s) 2021

\begin{abstract}
Background There is a paucity of epidemiological data regarding pesticide intoxication-associated acute kidney injury (AKI). Therefore, the aim of this study was to identify the epidemiological features, risk factors, and adverse outcomes of AKI in this population.

Methods The data used in this multi-center, hospitalized population-based, retrospective study were retrieved from electronic medical records. AKI was defined as an acute increase in serum creatinine according to the criteria of Kidney Disease: Improving Global Outcomes. The Charlson Comorbidity Index was used to evaluate the burden of in-hospital mortality. Results Of 3,371 adult patients in 11 hospitals, 398 (11.8\%) were diagnosed with AKI (grade 1, 218 [6.5\%]; grade 2, 89 [2.6\%]; grade 3, 91 [2.7\%]). Herbicide intoxication was associated with the highest incidence of AKI (53.5\%) and higher grades of AKI. After multivariable adjustment, pesticide categories and moderate or severe renal disease were independently associated with AKI. As compared with the referred category, insecticide and herbicide intoxications were associated with a 1.3-fold (95\% CI 1.688-3.245) and 3.8-fold (95\% CI3.537-6.586) greater risk of AKI. Regardless of the pesticide category, AKI was independently associated with in-hospital mortality, with odds ratios of 3.433 (95\% CI 1.436-8.203) for insecticides, 2.153 (95\% CI 1.377-3.367) for herbicides, and 4.524 (95\% CI 1.230-16.632) for unclassified or other pesticides.

Conclusion AKI is common in pesticide intoxication and associated with an increased in-hospital mortality. Herbicides pose the greatest risks of AKI and death.
\end{abstract}

Keywords Acute kidney injury $\cdot$ Pesticide $\cdot$ Epidemiology $\cdot$ Risk factor $\cdot$ Adverse outcome

\section{Introduction}

As a global public health concern, acute kidney injury (AKI) is not only associated with in-hospital adverse outcomes, but also increased short- and long-term risks of cardiovascular disease, chronic kidney disease, and mortality [1]. The International Society of Nephrology proposed the 0by 25 program that aims to prevent all avoidable deaths due to AKI by the year 2025 [2]. Although the worldwide incidence of AKI

Min He and Yanhua Wu contributed equally to this work.

Yuanhan Chen

chenyuanhan@gdph.org.cn

Xinling Liang

xinlingliang_ggh@163.com

Extended author information available on the last page of the article continues to increase, the epidemiology and treatment outcomes differ by regions and economic factors [3, 4]. The implementation of measures for the prevention of AKI is a major challenge in low-resource and low-income regions.

Pesticides, including herbicides, insecticides (which may include insect growth regulators, termiticides, etc.), nematicides, molluscicides, piscicides, avicides, rodenticides, bactericides, insect repellents, animal repellents, antimicrobials, and fungicides, are chemical compounds that are meant to control pests. Pesticides are widely used to promote economic development of agriculture-dependent countries in the Asia-Pacific region. However, the application of pesticides also threatens human health. Pesticide exposure can cause injury to the skin, as well as the nervous, reproductive, gastrointestinal, and urinary systems. Epidemiological studies have reported that long-term pesticide exposure is associated with chronic renal injury [5, 6]. For example, 
the Agricultural Health Study, a prospective study of cancer and other health outcomes, found that licensed pesticide applicators and their spouses are at higher risk of end-stage renal disease [7, 8]. Pesticide applicators in the Asia-Pacific region are also at greater risks of social problems, especially suicide [9-11]. A report by the World Health Organization estimated that pesticides were associated with 186,000 preventable suicides and 4.42 million disability-adjusted life years annually [12].

There is a paucity of epidemiological data of pesticide intoxication-associated AKI. According to a recent report based on the Taiwan Health Insurance Research Database, organophosphate intoxication was associated with a longterm higher risk of AKI [13]. Considering the acute nature of pesticide intoxication, the short-term effects on AKI also poses risk to human health. In addition, only organophosphate intoxication was investigated in the Taiwan study, thus the effects of different categories of pesticides should be further compared.

Therefore, the aim of the present multi-center retrospective study was to determine the epidemiological features, risk factors, and short-term adverse outcomes of adult patients with pesticide intoxication-associated AKI.

\section{Methods}

\section{Study design and data sources}

The China Collaborative Study on Acute Kidney Injury (CCS-AKI), sponsored by the Guangdong Provincial People's Hospital (Guangzhou, Guangdong Province, China), was a multi-center, hospitalized population-based, retrospective study designed to identify the epidemiological features of AKI in various regions and clinical settings. Demographic characteristics, diagnoses, and AKI-related laboratory test results were derived from electronic medical records. Creatinine measurements were adjusted to account for intrahospital differences. The CCS-AKI included 19 hospitals.

The CCS-AKI is registered with the clinicaltrials.gov website under registration number NCT03054142. The study protocol was approved by the Ethics Research Committee of Guangdong Provincial People's Hospital (approval no. GDREC2016327H) and conducted in accordance with the ethical principles for medical research involving human subjects as stated in the Declaration of Helsinki.

\section{Clinical definitions}

AKI was defined as an increase in serum creatinine of 0.3 $\mathrm{mg} / \mathrm{dL}$ within $48 \mathrm{~h}$ or a $50 \%$ increase from baseline within 7 days based on the criteria of Kidney Disease: Improving Global Outcomes [14]. The stage of AKI was determined using the peak creatinine level after AKI onset. Pesticides were classified into four categories: insecticides, herbicides, rodenticides, and unclassified or others.

The Charlson Comorbidity Index (CCI) was used to evaluate the burden of in-hospital mortality. Because the International Classification of Disease Code for CCS-AKI was not standardized among the participating hospitals, the CCI was calculated based on the diagnosis at discharge retrieved from electronic medical records [15]. According to the CCI definition, serum creatinine levels of $>3 \mathrm{mg} / \mathrm{dL}$ is regarded as a marker of moderate to severe renal disease [16].

\section{Statistical analyses}

Non-normally distributed continuous variables are presented as medians (25th and 75 th percentiles). Count data are expressed as the number of cases (\%), and the difference between groups was compared using the Chi-squared test or Fisher's exact test, as appropriate. The Bonferroni test was used for multiple comparisons of pesticide categories and AKI grades. Conditional multivariable logistic regression models were used to identify independent associations among variables. The associated $95 \%$ confidence intervals (CIs) were estimated. An interaction term was applied to estimate the effect of the pesticide category on AKI-associated mortality. If there was an interactive effect between the pesticide category and mortality, subgroup analysis was performed. All statistical analyses were performed using IBM SPSS Statistics for Windows, version 24.0. (IBM Corporation, Armonk, NY, USA). A two-tailed probability ( $p$ ) value of $<0.05$ was considered statistically significant.

\section{Results}

\section{Clinical characteristics}

The medical records of 3839 patients in 11 hospitals were retrieved from the CCS-AKI database, which included 50 cases of pesticide intoxication. According to the Institute of Geographic Sciences and Natural Resource Research (Chinese Academy of Sciences, Beijing, China), these 11 hospitals cover five of nine agricultural regions in China [17], including Southern China, the Yunnan-Guizhou Plateau, Northern arid and semiarid region, Sichuan Basin and surrounding regions, and Middle-lower Yangtze Plain. After exclusion of patients aged $<18$ years, 3371 adult patients (mean age 41 years; age range 28-54 years; 1470 [43.6\%] males) were included in this study. Among them, 1214 (36.0\%), 989 (29.3\%), 512 (15.2\%), and 598 (17.7\%) were treated for intoxication with insecticides, herbicides, rodenticides, and unclassified or other pesticides, respectively, and $58(1.7 \%)$ were treated for exposure to multiple pesticides. 
AKI was detected in 398 (11.8\%) patients (grade 1, 218 [6.5\%]; grade 2, 89 [2.6\%]; grade 3, 91 [2.7\%]). Compared with those without AKI, patients with AKI had higher rates of peripheral vascular disease, cerebrovascular disease, moderate or severe renal disease, and liver disease, as well as higher CCI scores (Table 1).

\section{AKI in different pesticide categories}

Among the hospitalized patients, the highest incidence of AKI (53.5\%) was associated with herbicide intoxication, following by insecticide intoxication (Table 1). The relationships among pesticide categories and AKI severity were further analyzed. Because of the limited number of cases, analysis was limited to the categories of insecticides, herbicides, and rodenticides. Besides the highest incidence, herbicide intoxication was associated with higher grades of AKI. For intoxication with herbicides, insecticides, and other pesticides, the rates of grade $2 \mathrm{AKI}$ were $5.7 \%, 1.6 \%$ and $1.1 \%$, and the rates of grade 3 were $7.4 \%, 1.1 \%$ and $0.4 \%$, respectively (Fig. 1a). A total of 103 patients (3.1\%) underwent renal replacement therapy (RRT). The rates of RRT were comparable among patients poisoned by insecticides, herbicides, and rodenticides $(3.5 \%, 3.6 \%$, and $2.1 \%$, respectively, $p=0.078$ ) (Fig. 1b).

\section{Risk factors for AKI}

To assess the risk factors for AKI, baseline variables that were considered clinically relevant or with a univariate relationship with AKI were entered into a multivariable logistic regression model (in stepwise forward conditional mode).
Table 1 Clinical characteristics by acute kidney injury

\begin{tabular}{|c|c|c|c|c|}
\hline & Non-AKI & AKI & $\chi^{2} / \mathrm{U}$ & $p$ value \\
\hline Gender, male & $1292(43.5 \%)$ & $177(44.5 \%)$ & 0.143 & 0.706 \\
\hline Age, years & $41(29,54)$ & $40(28,55)$ & -0.342 & 0.733 \\
\hline Pesticides categories & & & 147.053 & $<0.001$ \\
\hline Insecticides & $1087(89.5 \%)$ & $127(10.5 \%)$ & & \\
\hline Herbicides & $775(78.4 \%)$ & $213(21.6 \%)$ & & \\
\hline Rodenticides & $492(96.1 \%)$ & $20(3.9 \%)$ & & \\
\hline Other or unclassified & $566(94.6 \%)$ & $32(5.4 \%)$ & & \\
\hline Multiple & $52(89.7 \%)$ & $6(10.3 \%)$ & & \\
\hline \multicolumn{5}{|l|}{ Comorbidities } \\
\hline Myocardial infarction & $5(0.2 \%)$ & $2(0.5 \%)$ & 1.892 & 0.169 \\
\hline Congestive heart failure & $95(3.2 \%)$ & $8(2 \%)$ & 1.668 & 0.197 \\
\hline Peripheral vascular disease & $56(1.9 \%)$ & $14(3.5 \%)$ & 4.604 & 0.032 \\
\hline Cerebrovascular disease & $67(2.3 \%)$ & $16(4 \%)$ & 4.555 & 0.033 \\
\hline Dementia & $8(0.3 \%)$ & $2(0.5 \%)$ & & $0.334^{*}$ \\
\hline Chronic pulmonary disease & $47(1.6 \%)$ & $10(2.5 \%)$ & 1.83 & 0.176 \\
\hline Connective tissue disease & $16(0.5 \%)$ & $5(1.3 \%)$ & & $0.093^{*}$ \\
\hline Peptic ulcer disease & $13(0.4 \%)$ & $3(0.8 \%)$ & 0.743 & 0.389 \\
\hline Mild liver disease & $106(3.6 \%)$ & $24(6 \%)$ & 5.744 & 0.017 \\
\hline Diabetes without end-organ damage & $64(2.2 \%)$ & $11(2.8 \%)$ & 0.601 & 0.438 \\
\hline Hemiplegia & $2(0.1 \%)$ & $0(0 \%)$ & & $1^{*}$ \\
\hline Moderate or severe renal disease & $98(3.3 \%)$ & $81(20.4 \%)$ & 202.979 & $<0.001$ \\
\hline Diabetes with end-organ damage & $3(0.1 \%)$ & $1(0.3 \%)$ & & $0.395^{*}$ \\
\hline Tumor without metastasis & $26(0.9 \%)$ & $2(0.5 \%)$ & & $0.766^{*}$ \\
\hline Leukemia & $12(0.4 \%)$ & $1(0.3 \%)$ & & $1 *$ \\
\hline Lymphoma & $1(0 \%)$ & $0(0 \%)$ & & $1^{*}$ \\
\hline Moderate or severe liver disease & $4(0.1 \%)$ & $2(0.5 \%)$ & & $0.151^{*}$ \\
\hline Metastatic solid tumor & $42(1.4 \%)$ & $10(2.5 \%)$ & 2.792 & 0.095 \\
\hline AIDS & $3(0.1 \%)$ & $0(0 \%)$ & & $1 *$ \\
\hline Any liver disease & $107(3.6 \%)$ & $25(6.3 \%)$ & 6.704 & 0.01 \\
\hline Any diabetes & $65(2.2 \%)$ & $11(2.8 \%)$ & 0.53 & 0.467 \\
\hline Any malignant disease & $74(2.5 \%)$ & $13(3.3 \%)$ & & $0.398^{*}$ \\
\hline Score of Charlson Comorbidity Index & $0(0,0)$ & $0(0,2)$ & -9.484 & $<0.001$ \\
\hline
\end{tabular}

*The significance was calculated by Fisher's exact test 


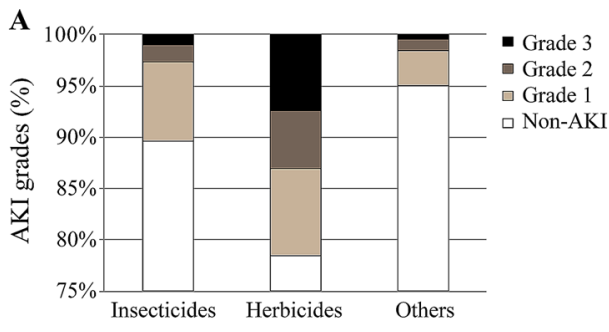

Fig. 1 AKI by pesticide categories. a AKI incidences and grades. Multiple comparisons among groups using Bonferroni correction: the incidence of AKI grade 2 or 3 was higher with herbicides than the other two groups, while comparable between the other two. b Rates

Table 2 Risk factors for AKI

\begin{tabular}{llll}
\hline & Odds ratio & $95 \%$ confidence interval & $p$ values \\
\hline Pesticides category & & & $<0.001$ \\
Insecticides & 2.340 & $1.688-3.245$ & $<0.001$ \\
Herbicides & 4.826 & $3.537-6.586$ & $<0.001$ \\
$\begin{array}{l}\text { Moderate or severe } \\
\text { renal disease }\end{array}$ & 6.490 & $4.658-9.042$ & $<0.001$ \\
\hline
\end{tabular}

The variables selected into multivariable logistic regression model for association analysis of AKI (in stepwise forward conditional mode) were pesticides category (non-insecticides and non-herbicides as reference), age, peripheral vascular disease, cerebrovascular disease, any liver disease and moderate or severe renal disease

These variables were pesticide categories (non-insecticides and non-herbicides as reference), age, peripheral vascular disease, cerebrovascular disease, any liver disease, and moderate or severe renal disease. After adjustment for multiple variables, pesticide categories and moderate or severe renal disease were independently associated with AKI development (Table 2). As compared with the referred category,

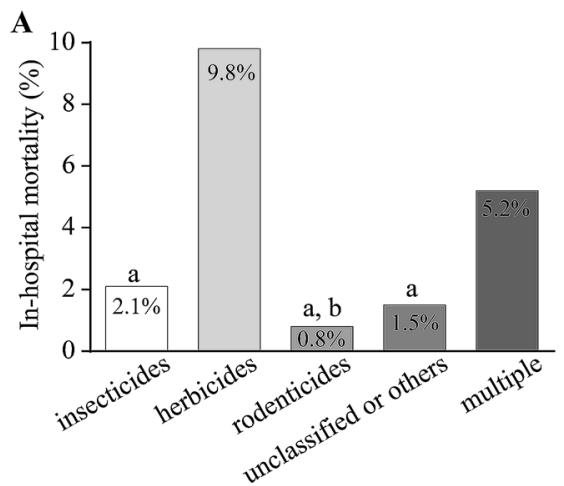

Fig. 2 In-hospital mortality in clinical settings. A The in-hospital mortality rate differed significantly among the different pesticide categories. a Significant difference vs. herbicides; b: significant difference vs. multiple pesticides. B The in-hospital mortality rate was

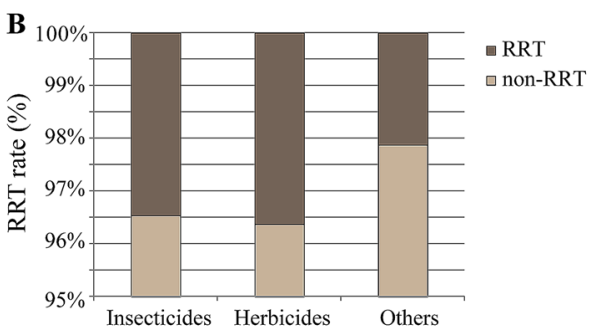

of renal replacement therapy. The rates of renal replacement therapy were comparable among the three groups $\left(\chi^{2} 5.112, p=0.078\right)$. AKI acute kidney injury, $R R T$ renal replacement therapy

insecticide and herbicide intoxications were associated with a 1.3-fold (95\% CI 1.688-3.245) and 3.8-fold (95\% CI3.537-6.586) greater risk of AKI, respectively.

\section{Association between AKI and in-hospital mortality}

A total of 139 patients (4.1\%) died during hospitalization. The raw in-hospital mortality rates for intoxications with herbicides, multiple pesticides, insecticides, unclassified or other pesticides, and rodenticides were $9.8 \%, 5.2 \%, 2.1 \%$, $1.5 \%$, and $0.8 \%$, respectively (Fig. 2a). After Bonferroni adjustment, the mortality rate associated with herbicides was higher than that of insecticides, unclassified or other pesticides, and rodenticides, and the mortality rate associated with multiple pesticides was higher than that of rodenticides. The mortality rates for non-AKI, and grades 1,2 , and $3 \mathrm{AKI}$ were $3.1 \%, 10.6 \%, 11.2 \%$, and $15.4 \%$, respectively. By Bonferroni adjustment for multiple comparison, the mortality rate was higher for all AKI grades than non-AKI. Although the results were not significant among the various grades of AKI, this increasing tendency indicated that the risk of

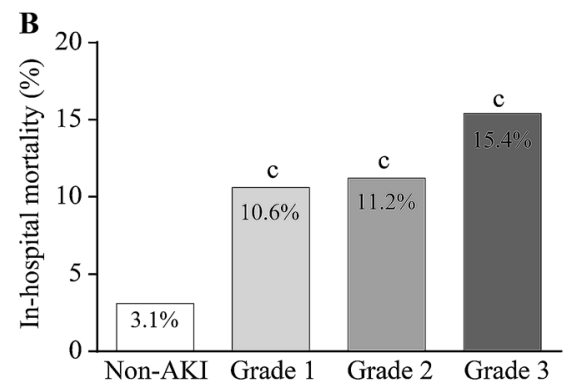

higher for any grade of AKI as compared with non-AKI. However, the mortality rates were comparable among various grades of AKI. Bonferroni adjustment was used for multiple comparisons. 
mortality gradually increased with a higher grade of AKI (Fig. 2b).

Because of an interaction between AKI and the pesticide category related to mortality in the preliminary analysis, the independent association of AKI with mortality was further assessed by stratification of the pesticide categories. Regardless of the category of pesticides, AKI was independently associated with in-hospital mortality, with odds ratios of 3.433 (95\% CI 1.436-8.203) for insecticides, 2.153 (95\% CI 1.377-3.367) for herbicides, and 4.524 (95\% CI 1.230-16.632) for unclassified or other pesticides (Table 3).

\section{Discussion}

The aim of this multi-center investigation was to identify the epidemiological features of pesticide intoxication-associated AKI. The results showed that the AKI was common in this population. Herbicides, the second most frequently applied pesticide, was associated with the highest risk and grade of AKI. After adjustment for multiple variables, herbicide intoxication was associated with a 3.8-fold greater risk of AKI. The in-hospital death rate was highest for herbicide intoxication. By stratification of pesticide categories, multivariable analysis identified an independent association of AKI with in-hospital mortality.

Pesticide intoxication remains an important public health concern, especially in Asia. According to national data of South Korea, the age-standardized rate of pesticide intoxication was 15.37 per 100,000 persons between 2006 and 2010, of which $57.3 \%$ of cases required hospitalization [18]. To the best of our acknowledgement, this is the first study to evaluate the epidemiological features of AKI in this population.

Table 3 Association between AKI and in-hospital mortality

\begin{tabular}{lcll}
\hline & Odds ratio & $95 \%$ CI & $p$ value \\
\hline Insecticides & & & \\
CCI & 1.223 & $1.007-1.485$ & 0.042 \\
University hospital & 12.091 & $1.617-90.425$ & 0.015 \\
$\quad$ AKI & 3.433 & $1.436-8.203$ & 0.006 \\
Herbicides & & & \\
$\quad$ University hospital & 6.247 & $1.948-20.033$ & 0.002 \\
AKI & 2.153 & $1.377-3.367$ & 0.001 \\
Other or unclassified pesticides & & \\
CCI & 1.458 & $1.167-1.822$ & 0.001 \\
AKI & 4.524 & $1.230-16.632$ & 0.023 \\
\hline
\end{tabular}

Variables of CCI, age, university hospital and AKI were selected into the multivariable logistic regression model in a forward conditional mode

$A K I$ acute kidney injury, 95\% CI 95\% confidential interval, $C C I$ Charlson Comorbidity Index
The raw incidence of AKI of $11.8 \%$ in this study was similar with the overall incidence of $11.6 \%$ in the EACH study (Epidemiology of AKI in Chinese Hospitalized Adults) [19]. However, the CCS-AKI criteria for AKI classification in the present study, which differed from those of the EACH study, strictly followed the definition of Kidney Disease: Improving Global Outcomes, whereas extended criteria were applied in the EACH study, which may have resulted in a higher incidence of AKI. A recent pilot study of CCS-AKI conducted by our group showed that the overall incidence of AKI in hospitalized adults was $8.2 \%$ and middle-aged patients were at the lowest risk of AKI [20]. Considering that most patients hospitalized for pesticide intoxication were middleaged, the risk of AKI in this population seemed to be higher than the overall hospitalized population. Notably, only hospitalized patients were included for analysis, thus the resulting epidemiological features in this study cannot necessarily be applied to other populations.

Although the detailed mechanisms have not been clarified, previous animal studies have demonstrated that pesticides can directly cause renal injury through reductions in metabolic functions and induction of oxidative stress [21-23]. Pesticide intoxication also manifests as gastrointestinal symptoms and gastric salvage further exacerbates fluid loss. Among the various categories of pesticides, herbicide intoxication is associated with greater risks of both AKI and mortality. From this perspective, herbicides are the most toxic. To the best of our knowledge, this study is the first to compare the renal toxic effects among various pesticide categories.

The strengths of this study include the large study sample. Thus, multiple comorbidities were adjusted for subgroup analysis to control for confounders. The use of multicenter data acquired from multi-level hospitals is another advantage. China is a large developing country that is going through industrial transformation from a typical agriculture country. Imbalances in economic and medical developments are evident across the nation. Thus, the epidemiological features of AKI associated with pesticide intoxication in China will provide valuable information for other developing countries. Among the 19 hospitals in the phase 1 database of CCS-AKI, four hospitals in first-tier cities (Beijing, Shanghai, Guangzhou, and Shenzhen) and four tertiary hospitals in capital cities (Hohhot, Urumqi, and Changchun) were excluded because of the low numbers of patients with pesticide intoxication, indicating that pesticide intoxication is not necessarily an important issue in developed industrial cities, but remains significant in low-resource agricultural regions.

Pesticide intoxication is an emergency condition. Although the diagnostic and therapeutic capabilities of local and non-university hospitals are often inadequate, as revealed by a recent survey [24], the benefit of referral to higher-level hospitals remains controversial. In this 
study, four of the 11 participating hospitals were provincelevel central hospitals and university hospitals. However, treatment in these hospitals was associated with a greater risk of death as compared with non-university hospitals (Table 3), suggesting that referral does not necessarily improve survival. This result might be attributed to the poorer condition of patients in central hospitals. While unavoidable delays for referral should also be considered, local availability of treatment is a reasonable strategy. Thus, improvement in local medical facilities is urgently needed.

Because of the retrospective study design, it was not possible to differentiate the indication of RRT for severe AKI, pesticide clearance, or multiple organ failure. Thus, a comparable rate of RRT (Fig. 1b) does not imply a similar rate of critical AKI among patients poisoned by different pesticides. Based on the finding that herbicide poisoning was associated with the highest rates of grade 2 and 3 AKI (Fig. 1a), herbicides seem to be the most detrimental pesticide with regard to AKI. Further, information regarding the exposure pathway and dose, severity of intoxication, and detoxification treatment was not available. In addition, it was not possible to determine whether the intoxication was self-induced, which is an important factor to improve survival. Thus, future prospective studies are needed to confirm these results.

In conclusion, AKI is a common complication of pesticide intoxication and associated with increased in-hospital mortality. Herbicides were associated with the highest risk of AKI and death.

Author contributions Each of the authors have made contributions to the current work. MH and YW devised the study plan and led the writing of the article. ZX, ZL, LH, GL, QH, YL, RG, HP, JX, XW, YZ, and $\mathrm{QH}$ collected the data. YC conducted the data analysis. XL supervised the entire process and gave constructive advice.

Funding This study was financially supported by the Medical Scientific Research Foundation of Guangdong Province (Grant No. A2019038), the Guangdong Science and Technology Project (Grant No. 2017A070709008), and the Chinese National Natural Science Foundation (Grant No. 81770667). Award for the Outstanding Young Medical Talents in Guangdong Province.

\section{Declarations}

Conflict of interest The authors have no competing interests to declare.

Ethics approval and consent to participate The study protocol was approved by the Ethics Management Committee of Guangdong Provincial People's Hospital (Approval No. GDREC.2016327H). This study was registered at ClincalTrials.gov (Registration No. NCT03054142). Because of the retrospective nature of this study, the need to obtain written informed consent was waived by the Ethics Management Committee of Guangdong Provincial People's Hospital.
Open Access This article is licensed under a Creative Commons Attribution 4.0 International License, which permits use, sharing, adaptation, distribution and reproduction in any medium or format, as long as you give appropriate credit to the original author(s) and the source, provide a link to the Creative Commons licence, and indicate if changes were made. The images or other third party material in this article are included in the article's Creative Commons licence, unless indicated otherwise in a credit line to the material. If material is not included in the article's Creative Commons licence and your intended use is not permitted by statutory regulation or exceeds the permitted use, you will need to obtain permission directly from the copyright holder. To view a copy of this licence, visit http://creativecommons.org/licenses/by/4.0/.

\section{References}

1. Chawla LS, Eggers PW, Star RA, Kimmel PL (2014) Acute kidney injury and chronic kidney disease as interconnected syndromes. N Engl J Med 371(1):58-66

2. Mehta RL, Cerdá J, Burdmann EA et al (2015) International Society of Nephrology's 0by25 initiative for acute kidney injury (zero preventable deaths by 2025): a human rights case for nephrology. Lancet 385(9987):2616-2643

3. Lameire NH, Bagga A, Cruz D et al (2013) Acute kidney injury: an increasing global concern. Lancet 382(9887):170-179

4. Hoste E, Kellum JA, Selby NM et al (2018) Global epidemiology and outcomes of acute kidney injury. Nat Rev Nephrol 14(10):607-625

5. Calvert GM (2016) Agricultural pesticide exposure and chronic kidney disease: new findings and more questions. Occup Environ Med 73(1):1-2

6. Valcke M, Levasseur ME, da Silva AS, Wesseling C (2017) Pesticide exposures and chronic kidney disease of unknown etiology: an epidemiologic review. Environ Health 16(1):49

7. Lebov JF, Engel LS, Richardson D, Hogan SL, Sandler DP, Hoppin JA (2015) Pesticide exposure and end-stage renal disease risk among wives of pesticide applicators in the Agricultural Health Study. Environ Res 143(Pt A):198-210

8. Lebov JF, Engel LS, Richardson D, Hogan SL, Hoppin JA, Sandler DP (2016) Pesticide use and risk of end-stage renal disease among licensed pesticide applicators in the Agricultural Health Study. Occup Environ Med 73(1):3-12

9. Page A, Liu S, Gunnell D et al (2017) Suicide by pesticide poisoning remains a priority for suicide prevention in China: analysis of national mortality trends 2006-2013. J Affect Disord 208:418-423

10. Bonvoisin T, Utyasheva L, Knipe D, Gunnell D, Eddleston M (2020) Suicide by pesticide poisoning in India: a review of pesticide regulations and their impact on suicide trends. BMC Public Health 20(1):251

11. Konradsen F, Dawson AH, Eddleston M, Gunnell D (2007) Pesticide self-poisoning: thinking outside the box. Lancet 369(9557):169-170

12. Guidelines for drinking-water quality, 4 th edn. https://www who.int/water_sanitation_health/publications/dwq-guidelines4/en/

13. Lee FY, Chen WK, Lin CL et al (2015) Organophosphate poisoning and subsequent acute kidney injury risk: a nationwide population-based cohort study. Medicine (Baltimore) 94(47):e2107

14. Kidney Disease: Improving Global Outcomes (KDIGO) AKI Work Group (2012) KDIGO clinical practice guideline for acute kidney injury. Kidney Int Suppl. 2(1):1-138

15. Mo L, Xie Z, Liu G et al (2020) Feasibility of coding-based Charlson comorbidity index for hospitalized patients in China, a representative developing country. BMC Health Serv Res 20(1):432 
16. Charlson ME, Pompei P, Ales KL, MacKenzie CR (1987) A new method of classifying prognostic comorbidity in longitudinal studies: development and validation. J Chronic Dis 40(5):373-383

17. http://www.resdc.cn/data.aspx? ?ATAID $=275$

18. Cha ES, Khang YH, Lee WJ (2014) Mortality from and incidence of pesticide poisoning in South Korea: findings from National Death and Health Utilization Data between 2006 and 2010. PLoS ONE 9(4):e95299

19. Xu X, Nie S, Liu Z et al (2015) Epidemiology and clinical correlates of AKI in Chinese hospitalized adults. Clin J Am Soc Nephrol 10(9):1510-1518

20. $\mathrm{Xu} \mathrm{L}, \mathrm{Wu} \mathrm{Y}, \mathrm{Chen} \mathrm{Y}$ et al (2021) Is acute kidney injury agedependent in the older adults: an observational study in two centers from North China. BMC Geriatr 21(1):7

21. Larsen K, Najle R, Lifschitz A, Virkel G (2012) Effects of sublethal exposure of rats to the herbicide glyphosate in drinking water: glutathione transferase enzyme activities, levels of reduced glutathione and lipid peroxidation in liver, kidneys and small intestine. Environ Toxicol Pharmacol 34(3):811-818
22. Tomita M, Okuyama T, Katsuyama H, Ishikawa T (2006) Paraquat-induced gene expression in rat kidney. Arch Toxicol 80(10):687-693

23. Mølck AM, Friis C (1997) The cytotoxic effect of paraquat to isolated renal proximal tubular segments from rabbits. Toxicology 122(1-2):123-132

24. Wu Y, Chen Y, Chen S et al (2018) Attitudes and practices of Chinese physicians regarding chronic kidney disease and acute kidney injury management: a questionnaire-based cross-sectional survey in secondary and tertiary hospitals. Int Urol Nephrol 55(11):2037-2042

Publisher's Note Springer Nature remains neutral with regard to jurisdictional claims in published maps and institutional affiliations.

\section{Authors and Affiliations}

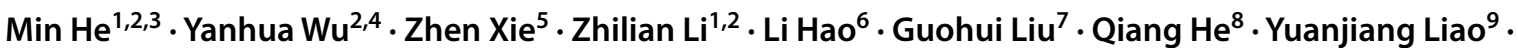
Rizwangul Ghappar ${ }^{10} \cdot$ Hongwei Peng $^{11}$. Jinglie Xie ${ }^{12} \cdot$ Xiaohong Wei $^{13} \cdot$ Yusheng Zhang ${ }^{14}$. Qiongyi Huang ${ }^{1}$. Yuanhan Chen ${ }^{1,2} \cdot$ Xinling Liang ${ }^{1,2}$ (1) on behalf of China collaborative study on AKI (CCS-AKI)

1 The Second School of Clinical Medicine, Southern Medical University, Guangzhou 510515, China

2 Division of Nephrology, Guangdong Provincial People's Hospital, Guangdong Academy of Medical Sciences, 106 Zhongshan Road 2, Guangzhou 510080, China

3 Division of Nephrology, Yue Bei People's Hospital Affiliated to Shantou University Medical College, Shaoguan 512026, China

4 Guangdong Provincial Geriatrics Institute, Guangzhou 510080, China

5 Department of Dermatology, Sichuan Provincial People's Hospital, University of Electronic Science and Technology of China, Chengdu 610072, China

6 Department of Nephrology, Second Hospital of Anhui Medical University, Hefei 230601, China

7 Department of Nephrology, Dongguan People's Hospital, Dongguan 523018, China
8 Department of Nephrology, Zhejiang Provincial People's Hospital (People's Hospital of Hangzhou Medical College), Hangzhou 310014, China

9 Department of Nephrology, Chongqing Ninth People's Hospital, Chongqing 400700, China

10 Department of Nephrology, First People's Hospital of Kashgar, Kashgar 844000, China

11 Department of Nephrology, People's Hospital of Wanning, The First Affiliated Hospital of Chongqing Medical University, Wanning 571500, China

12 Department of Nephrology, Lufeng People's Hospital, Shanwei 516500, China

13 Department of Nephrology, Chongzuo People's Hospital, Chongzuo 844000, China

14 Second Division of Internal Medicine, Wuhua People's Hospital, Meizhou 514400, China 